\title{
The combined effect of a novel vaccine for herd immunization on porcine respiratory and reproductive syndrome virus (PRRSV) and porcine parvovirus (PCVs) from Jinzhong-Shanxi of China
}

\author{
Longlong Zheng \\ Shanxi Agricultural University \\ Li Zhang (D912827159@qq.com) \\ Shanxi Agricultural University \\ Mingxue Zheng \\ Shanxi Agricultural University \\ Chen Wang \\ Shanxi Agricultural University \\ Huixin Kang \\ Shanxi Agricultural University \\ Fan Tan \\ Shanxi Agricultural University
}

\section{Research Article}

Keywords: PRRSV inactivated vaccine, NADC30-like, PCV2 VLPs vaccine, PCV3

Posted Date: March 7th, 2022

DOI: https://doi.org/10.21203/rs.3.rs-1381134/v1

License: @ (i) This work is licensed under a Creative Commons Attribution 4.0 International License. Read Full License 


\section{Abstract}

PRRSV and porcine circovirus (PCVs), two of the most significant swine diseases that affect the pig industry. NADC30-like or NADC34-like PRRSV and PCV3 has had a strong in China and could cause clinical disease on pigs. Co-infection of PRRSV and PCVs is frequently observed under field conditions and elicits more severe diseases. However, the endemic status of NADC30like/NADC34-like PRRSV and PCV3/PCV4 in Jinzhong of Shanxi is unclear under the field condition of immune inactivated vaccines or subunit vaccine (Virus-LikeParticles, VLPs). In this study, we examined 120 weaner dead pigs-lung tissue samples collected from a single PRRSV and PCV positive pig farm in Jinzhong-Shanxi from January to November 2021. Six NADC30like PRRSV and PCV3 strains were discovered in samples, and PRRSV NSP2 and ORF5 genomes \& PCV3 Cap genomes of these strains were sequenced. Phylogenetic analysis indicated that these novel PRRSV and PCV strains belong to NADC30-like PRRSV and PCV3, forming one group in Shanxi, Jinzhong. After NADC30-like PRRSV pregnant sows (African swine fever infection pressure) were immunized with Ch-1a inactivated vaccine for one year, NADC34-like PRRSV was not identified, and NADC30-like PRRSV was still an epidemic strain in the piglet herd. Importantly, PCV2 pregnant sows were immunized with subunit (Cap protein) vaccine for one year, PCV-4 was not detected, and PCV-3 was still an epidemic strain in the piglet herd. Immunization of sows with PRRSV inactivated vaccine and circular VLPs vaccine can reduce viremia and increase the survival rate of piglets, but cannot provide complete virological protection.

\section{Introduction}

Co-infection of PRRSV and PCVs is commonly observed under field conditions and elicits more severe diseases. The coinfection of PRRSV and PCVs (PCV2 and PCV3) might cause diseases even when PRRSV plays a limited role in the pathogenicity of the co-infection [1]. PRRSV can be regulated to a certain extent with macrolide drugs (Tilmicosin and Tyvanectin). PCV3 is associated with several clinical signs called porcine circovirus-associated diseases (PCVAD). There are still no effective drugs to prevent and control PCVs, and they can cause clinical diseases alone, and the economic loss is getting higher and higher. Vaccination is the best way to avoid economic losses from PRRSV and PCVs. In China, although various commercial attenuated PRRSV-live vaccines, PRRSV and PCVs-inactivated vaccines and PCVs-subunit vaccines have been widely utilized, PRRS and PCVs are still severe in the pig industry.

The modified-live virus (MLV) vaccines provide extremely limited cross-protection efficacy against the NADC30-like virus CHsx1401 infection [2], the NADC30-like strain HNhx [3]. Also, the inactivated vaccines revealed partial protection HP-PRRSV QH-08 Strain [4] and maternal inactivated vaccines vaccination can improve protection of pre-weaning piglets against PRRSV that transferring neutralizing antibodies to piglets [5]. As a result, maternal-derived immunity is a critical component for the survival and success of offspring in pigs to protect from circulating pathogens such as PRRSV and PCVs.

Moreover, the genetic diversity and complexity of PRRSV were further increasing. Constructed the global classification system of PRRSV based on the comprehensive analysis of the complete ORF5 gene sequence [6]. Since 2013, PRRS became prevalent again in China caused by new PRRSV variants, NADC34-like strains, which are considered to be imported from North American and adapted in China. NADC30-like or NADC34-like PRRSV virus strains transmitted quickly around herds in China although massive vaccination with all the commercial vaccines. Animal challenge study showed that the novel NADC30-like PRRSV SD17-36 isolate is low pathogenic [1] and CHsx1401 [7] \& HB17A [8] exhibits intermediate virulence.

Therefore, this study aimed to assess the positivity rates of PCV3/PCV4 and NADC30-Like/ NADC34-Like PRRSV in a largescale pig farm in Jinzhong-Shanxi Province, under immunity PRRSV CH1a and PCV2 VLPs vaccines during 1 year period.

\section{Materials And Methods}

\section{Sample collection}

Lung tissue samples ( $n=120 ; 1.5-2$ month-old pigs) were collected from one pig farm was major large-scale farm in Shanxi Province, which was distributed in Jinzhong city of Shanxi Province, accounting for about $3 \%$ of the total nursery stage. The 
samples were collected between January and November in 2021. Lung tissues were randomly selected from the respiratory syndrome clinical symptoms and anatomical dead, put into a sterile bag containing tissue protection solution, placed in an icebox, transported back to the laboratory for RT-PCR or PCR and sequencing analysis.

\section{RNA/DNA isolation and polymerase chain reaction (PCR)}

RNA/DNA were extracted from the collected tissue samples using the TRIzol®(TaKaRa, Dalian, China) and the EasyPure® Genomic DNA Kit (TransGen Biotech, Beijing, China) according to the manufacturer's instructions. cDNA was generated using a PrimeScriptTM RT reagent Kit (TaKaRa, Dalian, China) following the supplier's guidelines. PCV3 with PRRSV were detected using reverse transcription (RT)-PCR and PCR. The primers (PRRSV-F: 5'-TTGATTGGGATGTTGTGCTTC-3', PRRSV-R: 5'-

CAATGATGGCTTGAGCTGAGT-3') were designed based on the NSP2 region, and the sizes of the final amplicons were $628 \mathrm{bp}$ (NADC30-like PRRSV), 931 bp (HP-PRRSV), and 1,021 bp (typical PRRSV). This yielded a 603-bp PCR product containing a complete ORF5 gene of PRRSV [9]. The PCV3 specific primers designated as PCV3-F: 5'-TAGTATTACCCGGCACCTCGGAACC 3', and PCV3-R: 5'- ACAGGTAAACGCCCTCGCATGTGGG - 3', which amplified 649-bp [10]. The cycling conditions were: $95^{\circ} \mathrm{C}$ for $5 \mathrm{~min}$, followed by 35 cycles of $95^{\circ} \mathrm{C}$ for $30 \mathrm{~s}, 57^{\circ} \mathrm{C}$ for $30 \mathrm{~s}, 72^{\circ} \mathrm{C}$ for $35 \mathrm{~s}$, and a final extension at $72^{\circ} \mathrm{C}$ for $7 \mathrm{~min}$.

The PCR products were analysed using gel electrophoresis on a 1.5\% agarose gel in Tris-acetic-acid-EDTA buffer and stained with Ethidium Bromide (Ruitaibio, Beijing, China). The genome of the six of NADC30-like PRRSV and PCV3 strains (in this study) were registered in

National Microbiology Data Center(NMDC) No. shown Table 1. 
Table 1

Detection of NADC30-like PRRSV and PCV3 strains from Jinzhong-Shanxi in January. 2021 to November. 2021

\begin{tabular}{|c|c|c|c|c|c|c|}
\hline \multirow{2}{*}{$\begin{array}{l}\text { Name of } \\
\text { sample/ } \\
\text { Specimens }\end{array}$} & \multirow{2}{*}{$\begin{array}{l}\text { Clinical } \\
\text { symptoms }\end{array}$} & \multirow{2}{*}{$\begin{array}{l}\text { Pig } \\
\text { group* }\end{array}$} & \multirow[t]{2}{*}{ Collection date } & \multicolumn{3}{|l|}{ NMDC No. } \\
\hline & & & & PRRSV NSP2 & PRRSV ORF5 & PCV3 \\
\hline $\begin{array}{l}\text { SXJZ- } \\
\text { 2021- } \\
\text { 01/Lung }\end{array}$ & $\begin{array}{l}\text { Persistent } \\
\text { diarrhea, } \\
\text { Wasting, } \\
\text { Papular } \\
\text { dermatitis, } \\
\text { Respiratory } \\
\text { disorders, } \\
\text { Hind Limb } \\
\text { Paralysis, } \\
\text { Death }\end{array}$ & Weaner & 10.January.2021 & NMDCN0000R26 & NMDCN0000R2E & NMDCN0000R5U \\
\hline $\begin{array}{l}\text { SXJZ- } \\
\text { 2021- } \\
\text { 02/Lung }\end{array}$ & $\begin{array}{l}\text { Intermittent } \\
\text { diarrhea, } \\
\text { Wasting, } \\
\text { Papular } \\
\text { dermatitis } \\
\text {,Respiratory } \\
\text { disorders, } \\
\text { Hind Limb } \\
\text { Paralysis, } \\
\text { Death }\end{array}$ & Weaner & 12.March.2021 & NMDCN0000R27 & NMDCN0000R2D & NMDCN0000R5V \\
\hline $\begin{array}{l}\text { SXJZ- } \\
\text { 2021- } \\
\text { 03/Lung }\end{array}$ & $\begin{array}{l}\text { Intermittent } \\
\text { diarrhea, } \\
\text { Wasting, } \\
\text { Respiratory } \\
\text { disorders, } \\
\text { Hind Limb } \\
\text { Paralysis, } \\
\text { Death }\end{array}$ & Weaner & 11.May.2021 & NMDCN0000R28 & NMDCN0000R2C & NMDCN0000R60 \\
\hline $\begin{array}{l}\text { SXJZ- } \\
\text { 2021- } \\
04 / \text { Lung }\end{array}$ & $\begin{array}{l}\text { Intermittent } \\
\text { diarrhea, } \\
\text { Wasting, } \\
\text { Respiratory } \\
\text { disorders, } \\
\text { Hind Limb } \\
\text { Paralysis, } \\
\text { Death }\end{array}$ & Weaner & 13.July.2021 & NMDCN0000R29 & NMDCN0000R2B & NMDCN0000R64 \\
\hline $\begin{array}{l}\text { SXJZ- } \\
\text { 2021- } \\
05 / \text { Lung }\end{array}$ & $\begin{array}{l}\text { Intermittent } \\
\text { diarrhea, } \\
\text { Wasting, } \\
\text { Respiratory } \\
\text { disorders, } \\
\text { Hind Limb } \\
\text { Paralysis, } \\
\text { Death }\end{array}$ & Weaner & 10.September.2021 & NMDCN0000R2G & NMDCN0000R2A & NMDCN0000R65 \\
\hline $\begin{array}{l}\text { SXJZ- } \\
\text { 2021- } \\
\text { 06/Lung }\end{array}$ & $\begin{array}{l}\text { Intermittent } \\
\text { diarrhea, } \\
\text { Wasting, } \\
\text { Respiratory } \\
\text { disorders, } \\
\text { Hind Limb } \\
\text { Paralysis, } \\
\text { Death }\end{array}$ & Weaner & 12.November.2021 & NMDCN0000R2F & NMDCN0000R29 & NMDCN0000R67 \\
\hline
\end{tabular}

*Samples were classified into six groups of time, suckling pigs (<30 days), weaner (30-60 days), grower (60-90 days) and finisher ( $\geq 90$ days) 


\section{Genome sequencing}

Further, the purified RT-PCR or PCR products were sequenced by Sangon Biological Technology (Beijing, China).

\section{Genome alignment and phylogenetic analysis}

The obtained full-length genomic sequences were assembled using the SeqMan program of DNAstar software, version 7.0 (DNASTAR Inc., Madison, WI, USA). Further, the sequences of PRRSV NSP2/ORF5 and PCV3 Cap and deduced proteins were analyzed by the EditSeq and MegAlign programs of DNAstar (DNASTAR Inc., Madison, WI, USA). Next, phylogenetic analysis was performed based on the nucleotide sequences, generated by the distance-based neighbor-joining method using the Molecular Evolutionary Genetics Analysis 6 (MEGA 6) software (www.megasoftware.net). Finally, the bootstrap values of the phylogenetic tree were evaluated with 1000 replicates. PRRSV and PCVs strains used in our study of phylogenetic trees shown Table 2, Table 3 .

Table 2

PRRSV strains used in our study of phylogenetic trees

\begin{tabular}{|lllll|}
\hline No. & Strain/Genotype & Country & Year & GenBank No. \\
\hline 1 & CH-1a/Typical & China & 1996 & AY032626 \\
\hline 2 & VR2332/Typical & USA & 2002 & AF535152 \\
\hline 3 & R98/Typical & China & 2006 & DQ355796 \\
\hline 4 & CH-1R/Typical & China & 2008 & EU807840 \\
\hline 5 & WUH6/Typical & China & 2016 & KU523367 \\
\hline 6 & JXA1-R/HP-PRRSV & China & 2020 & MT163314 \\
\hline 7 & TJM-F92/HP-PRRSV & China & 2020 & MN508255 \\
\hline 8 & HUN4/HP-PRRSV & China & 2016 & EF635006 \\
\hline 10 & GDr180/HP-PRRSV & China & 2018 & MG972942 \\
\hline 11 & NADC30-USA-2012 & USA & 2012 & JN654459 \\
\hline 12 & CHsx1401/NADC30-link & China & 2014 & KP861625 \\
\hline 13 & SD17-36/NADC30-link & China & 2018 & MH121061 \\
\hline 14 & Guangdong/NADC34-link & China & 2017 & LNWK96 \\
\hline 15 & Guangdong/NADC34-link & China & 2017 & LNWK130 \\
\hline 16 & HNLCL82-1811/NADC34-link & China & 2018 & MN648057 \\
\hline 17 & RFLP 1-4-4 & USA & 2020 & MW887655 \\
\hline 18 & P129/Typical & USA & 2002 & AF494042 \\
\hline 19 & GM2 & China & 2011 & JN662424 \\
\hline 20 & SX2009 & China & 2009 & FJ895329 \\
\hline
\end{tabular}


Table 3

PCVs strains used in our study of phylogenetic trees

\begin{tabular}{|lllll|}
\hline No. & Strain/ Genotype & Country & Year & Accession \\
\hline 1 & PCV1 & China & 2015 & KP337349 \\
\hline 2 & PCV1-Qu & Canada & 2021 & MK872393 \\
\hline 3 & ZJ/C/PCV2 & China & 2004 & AY686764 \\
\hline 4 & DBN-SX01/PCV2 & China & 2006 & GQ404800 \\
\hline 5 & SH/PCV2 & China & 2006 & HM038027 \\
\hline 6 & DBN-SX07-2/PCV2 & China & 2007 & HM641752 \\
\hline 7 & LG/PCV2 & China & 2008 & HM038034 \\
\hline 8 & SXJZ/PCV2 & China & 2014 & KX068221 \\
\hline 9 & 14SX01/PCV2 & China & 2015 & KP975432 \\
\hline 10 & WH/PCV2 & China & 2015 & MK604497 \\
\hline 11 & CN-Shandong-2-201703/PCV3 & China & 2017 & KY778777 \\
\hline 12 & CN-HLJ-p6-2018/PCV3 & China & 2018 & MN431643 \\
\hline 13 & Pig-CN-HeNan170546-3/PCV3 & China & 2018 & MF769809 \\
\hline 14 & Shanxi-2018/PCV3 & China & 2018 & MH548436 \\
\hline 15 & SDA004-2018/PCV3 & China & 2018 & MK178296 \\
\hline 16 & CN-Xinjiang-AL15/PCV3 & China & 2018 & MK562413 \\
\hline 17 & Henan-2019/PCV4 & China & 2019 & MT002818 \\
\hline 18 & ZJ-459-20130424/PCV3 & China & 2019 & MK744559 \\
\hline 19 & Shanxi-2020/PCV3 & China & 2020 & MZ449243 \\
\hline 20 & GX2020/ PCV4 & China & 2020 & MT311854 \\
\hline & & & & \\
\hline
\end{tabular}

\section{STATISTICAL ANALYSIS}

Te statistical signifcance of the diferences was assessed by Student's t test and one-way ANOVA with multiple comparisons. Signifcant diferences in the survival curves were determined by log-rank analysis. A P value less than 0.05 was considered to indicate statistical signifcance, $\mathrm{P}<0.01$ was considered highly signifcant, $\mathrm{P}<0.001$ was considered very highly signifcant, and $\mathrm{P}<0.0001$ was considered extremely highly signifcant.

\section{Results}

\section{Changes in Herd Immunization Vaccines}

After long-term whole herd immunization with PRRSV inactivated vaccine and PCV2 VLPs vaccine, the mortality rate of pigs in the breeding period (12\% 5.5\%), PCVAD (15\% 8\%), PRRSVs PCR positive rate (45\% 10\%) and PCVs PCR positive rate (90\% 75\%) showed a significant weakening trend (Table 4). 
Table 4

Vaccine impact

\begin{tabular}{|lllll|}
\hline Collection date & $\begin{array}{l}\text { Mortality throughout the incubation } \\
\text { period }\end{array}$ & PCVAD & $\begin{array}{l}\text { PRRSV PCR positive } \\
\text { rate }\end{array}$ & $\begin{array}{l}\text { PCVs PCR positive } \\
\text { rate }\end{array}$ \\
\hline September.2020 & $12 \%$ & $15 \%$ & $45 \%$ & $90 \%$ \\
\hline January.2021 & $11.5 \%$ & $15 \%$ & $42 \%$ & $90 \%$ \\
\hline February.2021 & $9.5 \%$ & $13 \%$ & $40 \%$ & $85 \%$ \\
\hline Mar.2021 & $8.5 \%$ & $13 \%$ & $40 \%$ & $80 \%$ \\
\hline April.2021 & $8.5 \%$ & $12 \%$ & $35 \%$ & $80 \%$ \\
\hline May.2021 & $7 \%$ & $12 \%$ & $30 \%$ & $80 \%$ \\
\hline June.2021 & $7.5 \%$ & $10 \%$ & $25 \%$ & $75 \%$ \\
\hline July.2021 & $7.5 \%$ & $10 \%$ & $20 \%$ & $70 \%$ \\
\hline August.2021 & $7 \%$ & $10 \%$ & $20 \%$ & $70 \%$ \\
\hline September.2021 & $7 \%$ & $9 \%$ & $20 \%$ & $65 \%$ \\
\hline October.2021 & $6.2 \%$ & $9 \%$ & $25 \%$ & $70 \%$ \\
\hline November.2021 & $5.5 \%$ & $8 \%$ & $15 \%$ & $70 \%$ \\
\hline December.2021 & $5.5 \%$ & $8 \%$ & $10 \%$ & $65 \%$ \\
\hline
\end{tabular}

\section{NADC30-link NSP2 and ORF5 dataset-phylogenetic analysis}

In this study, we focus only on the PRRSV strains reduction. Safer inactivated PRRSV vaccines are used in the present investigation, whole pig herd 12 months in jinzhong, Shanxi, China. Moderate virulence strains(MLV-PRRSV) NADC30-like PRRSVs gradually became dominant strains, replacing highly pathogenic strains(HP-PRRSV). NADC30-like PRRSVs are undergoing a reduction in population genetic diversity in the farm by genetic evolution analysis of NSP2 and ORF5 gene of 6 isolates (FIGURE 1).

PCV3 Capsid dataset-phylogenetic analysis

PCV2 VLPs vaccine does not prevent vertical transmission of PCV3 within the pig farm and does not provide virological protection (FIGURE 2).

\section{Discussion}

PRRSV is highly prevalent in pig populations and is responsible for severe economic losses to the swine industry worldwide. The newly emerged lineage 1 PRRSVs (especially the NADC30-like and NADC34-like viruses) have posed a direct threat to the Chinese pig industry since 2013. Similarly, to other RNA viruses, PRRSV has the ability to continuously undergo genetic/antigenic changes [11]. PCV3 is a newly identified circovirus from swine in the USA in 2015 though metagenomic sequencing and may be associated with PDNS [12]. PCV3 may be a important pathogen in the swine industry, infection and the disease caused by PCV3, has been reported in many swine farms worldwide with high positive rates. The PCV3 infection can lead to respiratory diseases, digestive disorders, reproductive disorders, multisystemic inflammation, and immunosuppression [13].

In China, PCV3 was first reported in Guangdong Province in 2016 [14]. The first NADC34-like PRRSV in China was reported in Liaoning province in 2018, designated LNWK96 and LNWK130 [15]. After that, 20 more mildly pathogenic NADC34-like PRRSVs and 600 PCV3 were reported in Heilongjiang, Henan, and Fujian provinces $[16,17,18,19,20]$. Only one case of NADC30-like PRRSV CHsx1401 strain has been reported in Shanxi, and few epidemiological report on NADC34-like PRRSV and 
PCV3 strain has been made. Central Shanxi, mainly located in the basin area, has lush vegetation and low winds, making it an unfavourable environment for pathogen transmission [21]. The relatively stable climate is not conducive to the introduction of the virus and is an ideal place to explore the evolution of NADC34-like PRRSVs and PCV3 in pigs under immune conditions.

Vaccination is always the primary option to control and eradicate deadly diseases e.g., PRRS and PCVAD. Still, some inactivated/VLPs vaccines do not often protect the animals against PRRSV and PCV challenges when major antigenic shifts or novel virus subtypes appear. NADC30-like SXJZ-2021 strains show high genetic variations and incidence of recombination, compared with lineage 8 (MLV Ch-1a-like) and New Intro cluster (NADC30-like CHsx1401). PCV3 SXJZ-2021 strains show high genetic variations, compared with PCV2-b DBN-SX. These characteristics probably made current vaccines ineffective and confer PRRS and PCVAD much easier to escape the immune surveillance. Thus, they adapted well during the pig populations.

Vaccines are usually effective strategies for virus control. It is still a controversial question about the usage of PRRSV vaccines, achieved a certain level of clinical protection. There are no better choices currently under the circumstances of PRRSV pandemics [22]. Since the emergence of PRRSV, several different kinds of vaccines have been developed and widely adopted in the field, the most common of which are inactivated vaccines and live attenuated vaccines. However, inactivated vaccines against PRRSV cannot elicit a strong immune response, and live attenuated vaccines usually provide effective homologous protection but limited protection against heterologous strains, owing to the vast genetic diversity and high mutation rate of PRRSV [23].

But, inactivated vaccines are safer, more stable, and easier to store, higher antigen content and no ADE phenomenon compared with live vaccine. So, more and more inactivated vaccines are used in Chinese pig farms to replace live vaccines for immunization to prevent and control PRRS. These vaccines are effective in reducing clinical signs, decreasing viremia and shortening duration of viral shedding. They can provide an efficient protection against a lethal challenge with their respective parental HP-PRRSV isolates. We must clearly understand any one vaccine can not completely prevent infection and establish sterilizing immunity, etc. PRRSV and PCVs.

\section{Conclusions}

This study was the first detailed investigation into epidemiology of PCV3 and NADC30-like PRRSV under the field condition of immune PRRSV inactivated vaccine and PCV2 VLPs vaccine for 1 year in Jinzhong, Shanxi Province, China. During the 1-year surveillance period, the inactivated PRRSVVaccine and PCV2 VLPs vaccine could not provide virological protection to the piglets, and the piglets were still getting sick. In view of the continuous variation of PRRSVs and PCVs virus genes, it is necessary to provide better immune adjuvants for obtaining better clinical immune protection.

\section{Abbreviations}

PCVs

Porcine circovirus

PRRSVs

Porcine reproductive and respiratory syndrome viruses

PRRSV

Porcine respiratory and reproductive syndrome virus

PCV1

Porcine circovirus type 1

PCV2

Porcine circovirus type 2

PCV3

Porcine circovirus type 3

PCR 
Polymerase chain reaction

nt

Nucleotides

PNDS

Porcine dermatitis and nephropathy syndrome

ORF

Open reading frame

Rep

Replication-associated protein

Cap

Capsid

VLPS

Virus-LikeParticles.

\section{Declarations}

\section{Acknowledgments}

The authors would like to thank the staff at Shanxi Agricultural University (SAU; Taigu, China) who were directly involved in the production of their practical and professional assistance with vaccine management during the preparation of this review article.

\section{Authors'contributions}

M.X. Zheng and L. Zhang edited the review. L.L. Zheng completed data collection and experimentation, prepared the main manuscript text and Figures 1-2 of the paper. H.X. Kang, F. Tan and C. Wang prepared Table 1-4. All authors reviewed and considered the manuscript.

\section{Funding}

1 College of Veterinary Medicine Scientific research innovation project, Shanxi Agricultural University(DY-Q002); 2 Graduate education innovation project of Shanxi Province in 2021,China(2021Y316); 3 Financial reward for doctoral graduates and researchers from Shanxi Province to work in Shanxi, China(SXBYKY2021041); 4 Shanxi Province Basic Research Program, China(20210302124495)

\section{Availability of data and materials}

All data included in this study are available upon request by contact with the first author.

\section{Ethics approval and consent to participate}

This article did not include animal experiments.

\section{Consent for publication}

Not applicable.

\section{Competing interests}

None.

\section{References}


1. Zhou L, Yang B, Xu L, Jin H, Ge X, Guo X, Han J, Yang H. 2017. Efficacy evaluation of three modified-live virus vaccines against a strain of porcine reproductive and respiratory syndrome virus NADC30-like. Vet Microbiol.207:108-116.

2. Chen N, Li S, Ye M, Huang Y, Huang Y, Xiao Y, Yu X, Dong J, Tian K, Zhu J. 2019. A novel NADC30-like porcine reproductive and respiratory syndrome virus (PRRSV) plays a limited role in the pathogenicity of porcine circoviruses (PCV2 and PCV3) and PRRSV co-infection. Transbound Emerg Dis. 66(1):28-34.

3. Chen XX, Zhou X, Guo T, Qiao S, Guo Z, Li R, Jin Q, Hu X, Xing G, Deng R, Wan B, Zhang G. 2021. Efficacy of a live attenuated highly pathogenic PRRSV vaccine against a NADC30-like strain challenge: implications for ADE of PRRSV. BMC Vet Res. 17(1):260.

4. Ding Y, Wubshet AK, Ding X, Zhang Z, Li Q, Dai J, Hou Q, Hu Y, Zhang J. 2021. Evaluation of Four Commercial Vaccines for the Protection of Piglets against the Highly Pathogenic Porcine Reproductive and Respiratory Syndrome Virus (hp-PRRSV) QH-08 Strain. Vaccines (Basel). 9(9):1020.

5. Kick AR, Wolfe ZC, Amaral AF, Cortes LM, Almond GW, Crisci E, Gauger PC, Pittman J, Käser T. 2021. Maternal Autogenous Inactivated Virus Vaccination Boosts Immunity to PRRSV in Piglets. Vaccines (Basel). ;9(2):106.

6. Shi M, Holmes EC, Brar MS, Leung FC. 2013. Recombination is associated with an outbreak of novel highly pathogenic porcine reproductive and respiratory syndrome viruses in China. J Virol. 87(19):10904-7.

7. Bian T, Sun Y, Hao M, Zhou L, Ge X, Guo X, Han J, Yang H. 2017. A recombinant type 2 porcine reproductive and respiratory syndrome virus between NADC30-like and a MLV-like: Genetic characterization and pathogenicity for piglets. Infect Genet Evol. 2017 Oct;54:279-286.

8. Murtaugh M.P.; Stadejek T.; Abrahante J.E.; Lam T.T.; Leung F.C. 2010. The ever-expanding diversity of porcine reproductive and respiratory syndrome virus. Virus Res. 154, 18-30.

9. Sun YF, Yu H, Jiang X, Ma JF, Xu CQ, Yu XX, Li LA. 2020. Novel ORF5 deletion of NADC30-like porcine reproductive and respiratory syndrome viruses circulating in northern China from 2016 to 2018. J Vet Diagn Invest. 32(6):928-932.

10. Ku, X. , Chen, F. , Li, P. , Wang, Y. , Yu, X. , Fan, S. , Qian, P. , Wu, M. , \& He, Q. 2017. Identification and genetic characterization of porcine circovirus type 3 in China. Transboundary and Emerging Diseases, 64(3), 703-708.

11. Palinski R, Piñeyro P, Shang P, Yuan F, Guo R, Fang Y, Byers E, Hause BM. A Novel Porcine Circovirus Distantly Related to Known Circoviruses Is Associated with Porcine Dermatitis and Nephropathy Syndrome and Reproductive Failure. J Virol. 2016 Dec 16;91(1):e01879-16.

12. Phan TG, Giannitti F, Rossow S, Marthaler D, Knutson TP, Li L, Deng X, Resende T, Vannucci F, Delwart E. 2017. Detection of a novel circovirus PCV3 in pigs with cardiac and multi-systemic inflammation. Virol J. 2016 Nov 11;13(1):184.

13. Chen GH, Mai KJ, Zhou L, Wu RT, Tang XY, Wu JL, He LL, Lan T, Xie QM, Sun Y, Ma JY. 2017. Detection and genome sequencing of porcine circovirus 3 in neonatal pigs with congenital tremors in South China. Transbound Emerg Dis.64(6):1650-1654.

14. Zhang HL, Zhang WL, Xiang LR, Leng CL, Tian ZJ, Tang YD, Cai XH. 2018. Emergence of novel porcine reproductive and respiratory syndrome viruses (ORF5 RFLP 1-7-4 viruses) in China. Vet Microbiol. 222:105-108.

15. Song S, Xu H, Zhao J, Leng C, Xiang L, Li C, Fu J, Tang YD, Peng J, Wang Q, Zhao H, An T, Cai X, Zhang H, Tian ZJ. 2020. Pathogenicity of NADC34-like PRRSV HLJDZD32-1901 isolated in China. Vet Microbiol. 246:108727.

16. Liu, J., Wei, C., Lin, Z., Xia, W., Ma, Y., Dai, A., \& Yang, X. 2019. Full genome sequence analysis of a 1-7-4-like PRRSV strain in Fujian Province, China.Peer J, 7, e7859

17. Xie, C., Ha, Z., Nan, F., Zhang, Y., Zhang, H., Li, J., Zhang, P., Han, J., Zhuang, X., Zhang, J., Lu, H., \& Jin, N. (2020). Characterization of porcine reproductive and respiratory syndrome virus (ORF5 RFLP 1-7-4 viruses) in northern China. Microbial Pathogenesis, 140, 103941.

18. Xu, H., Song, S., Zhao, J., Leng, C., Fu, J., Li, C., Tang, Y. D., Xiang, L., Peng, J., Wang, Q., Zhao, H., An, T., Cai, X., Zhang, H., \& Tian, Z. J. 2020. A potential endemic strain in China: NADC34-like porcine reproductive and respiratory syndrome virus. Transboundary And Emerging Diseases, 67, 1730-1738. 
19. Bao H, Li X. 2021. Emergence and spread of NADC34-like PRRSV in China. 2021. Transbound Emerg Dis. 68(6):30053008.

20. Yue WD, LiuYH, Zhang XRg, Ma HL and He JP. 2021. Molecular detection of porcine circovirus type 3 in Shanxi Province, China. Animal Diseases.Animal Diseases.24(1)1-9.

21. Wang H, Xu Y, Feng W. 2021. Porcine Reproductive and Respiratory Syndrome Virus: Immune Escape and Application of Reverse Genetics in Attenuated Live Vaccine Development. Vaccines (Basel). 9(5):480.

22. Nan Y, Wu C, Gu G, Sun W, Zhang Y, Zhou E. 2017. Improved vaccine against PRRSV: current Progress and future perspective. Front Microbiol. 8:1635.

23. Sui X, Guo X, Jia H, Wang X, Lin W, Li M, Gao X, Wu J, Jiang Y, Willems L, Zhu H, Xin T, Hou S. 2018. Genomic sequence and virulence of a novel NADC30-like porcine reproductive and respiratory syndrome virus isolate from the Hebei province of China. Microb Pathog. 125:349-360.

\section{Figures}

NSP2

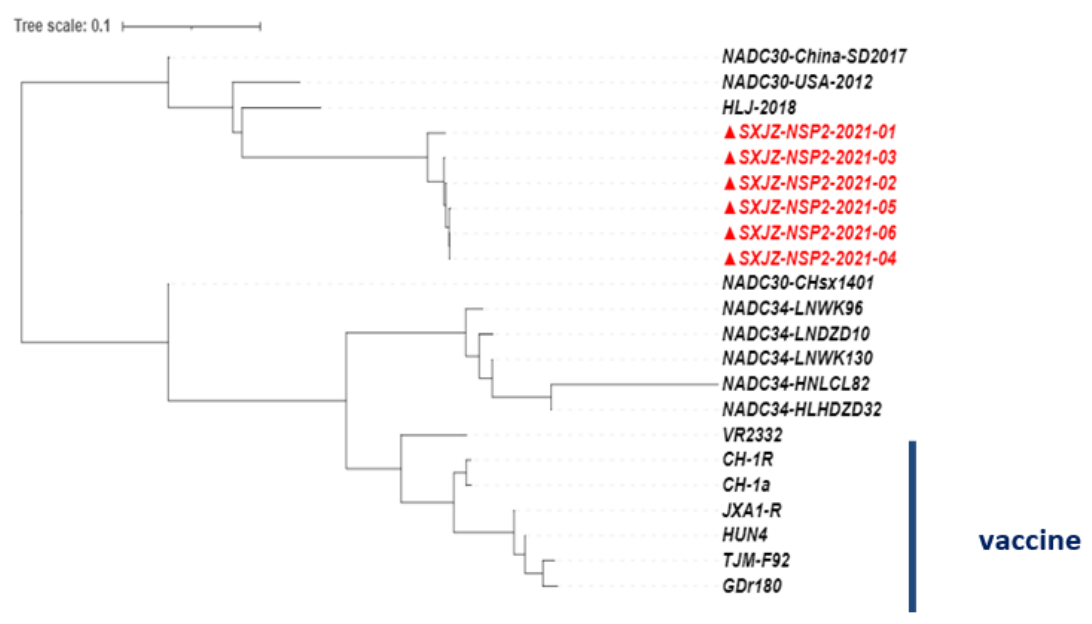

GP5

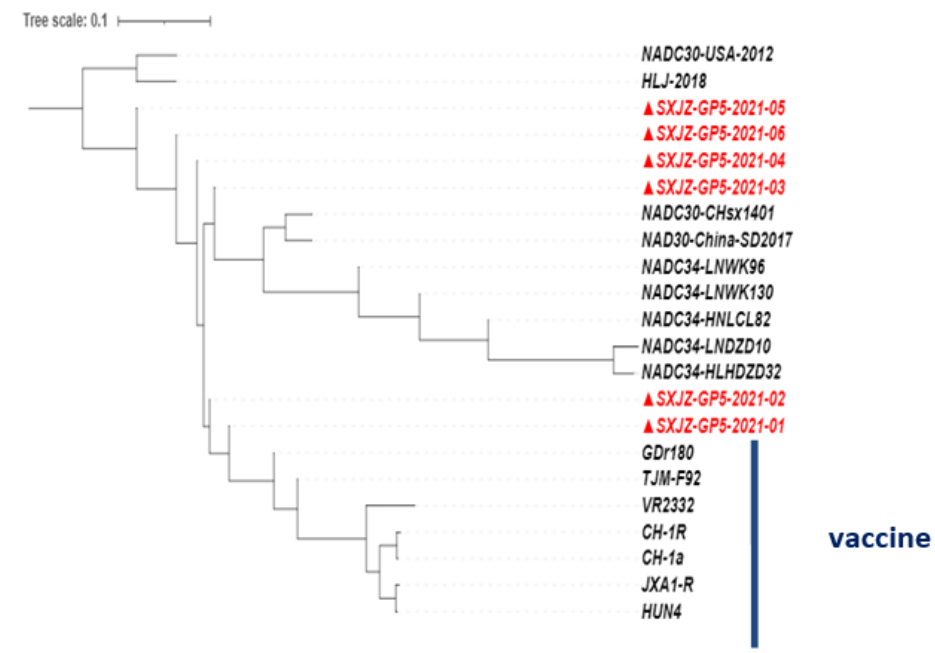


Figure 1

The genomic organization and phylogeny of NADC30-link PRRSV.

Cap

Tree scale: 1

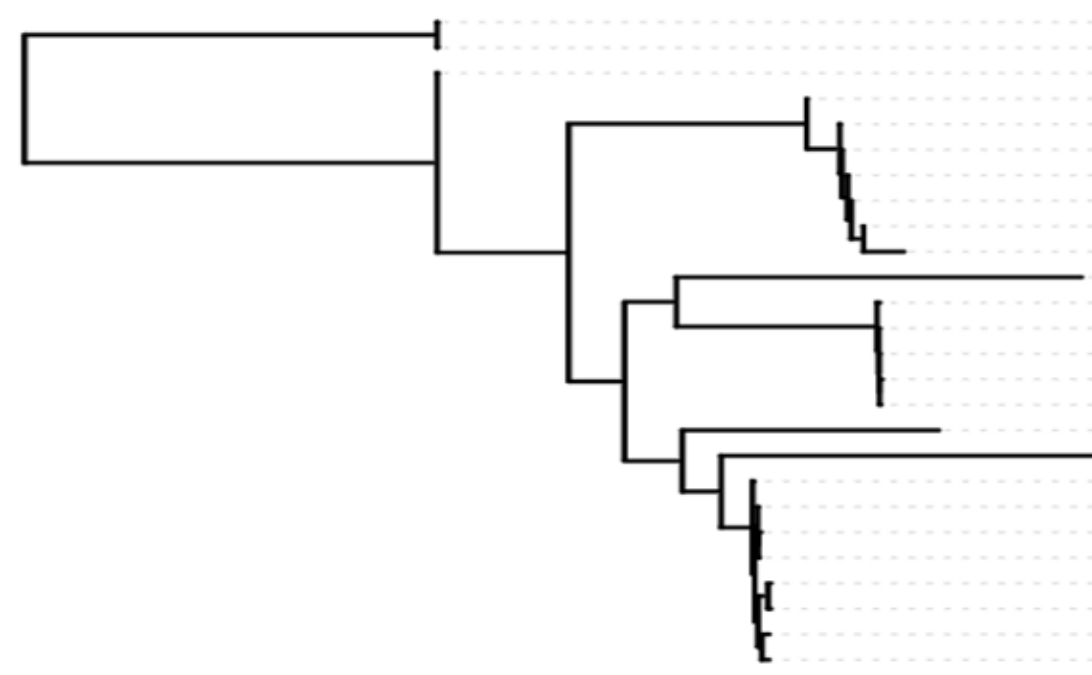

PCV1

PCV1-Qu

SXJZ-2021-01

PCV3-ZJ-459-20130424

SXJZ-2021-02

SXJZ-2021-03

SXJZ-2021-04

SXJZ-2021-05

SXJZ-2021-06

PCV3-CN-Xinjiang-AL15

PCV3-shanxi-2018

PCV3-Shanxi-2020

PCV3-HeNan170546-3

PCV3-SDA004

PCV3-HLJ-P6-2018

PCV3-CN-Shandong-2-201703

PCV4-GX2020-FCG49

PCV4-Henan-2019

PCV2-14SX01

PCV2-DBN-SX07

PCV2-SH

PCV2-ZJ-C

PCV2-DBN-sX01.seq

PCV2-SXJZ

PCV2-LG

Figure 2

The genomic organization and phylogeny of PCV3 Cap. 